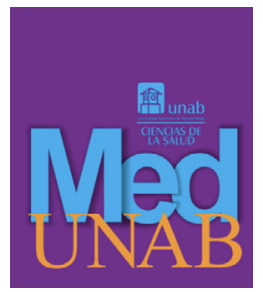

REVISTA DE LA FACULTAD

DE CIENCIAS DE LA SALUD

\title{
Transformando la enfermería a través el conocimiento: resultados del programa de guías de buenas prácticas de la Registered Nurses' Association of Ontario (RNAO)
}

\author{
Transforming nursing through knowledge: results of the Registered Nurses' \\ Association of Ontario (RNAO) good practice guidelines program \\ Transformando a enfermagem através do conhecimento: resultados do programa de \\ guia de boas práticas da Registered Nurses‘ Association of Ontario (RNAO).
}

Es un enorme placer acompañar una vez más, a través de esta editorial, a la revista MedUNAB, al equipo editorial y a su editora, Dra. Mary Luz Jaimes Valencia, para así mantenerlos al día en los progresos alcanzados con el programa de Guías de Buenas Prácticas de la Registered Nurses' Association of Ontario (RNAO).

El programa de Guías de Buenas Prácticas (BPG Program) fue lanzado en 1999 por la RNAO y fue financiado por el Ministerio de Salud de Ontario. El propósito de este programa, desde su inicio, ha sido apoyar a las enfermeras proporcionándoles guías basadas en la evidencia para el cuidado del paciente en diversos contextos y sectores de la salud. Con ya cincuenta y cuatro guías en inglés, y otros idiomas inclusive, en su mayoría traducidas al español, se facilita su aseso libre de costo a millones de enfermeras y demás profesionales sanitarios en todo el mundo (www.rnao.ca).

Un avance muy importante que ha tenido el programa RNAO de Guías de Buenas Prácticas es su sistema de datos online: Nursing Quality Indicators for Reporting and Evaluation (NQuIRE). A través de NQuIRE, RNAO recopila, analiza y trasmite datos sobre los indicadores de calidad enviados por los distintos servicios sanitarios. Además, NQuIRE ayuda a los centros BPSO a realizar mejoras efectivas en la práctica, ya que les proporciona datos organizados y comparativos sobre los procesos de cuidados orientados por las guías y los consiguientes resultados clínicos. Con los datos NQuIRE, los centros acreditados como Best Practice Spotlight Organizations (BPSO) pueden monitorear su progreso, identificar áreas de mejora continua, destacar áreas en las que debe profundizar la investigación y mejorar la calidad para optimizar los resultados clínicos, organizativos y del sistema sanitario (1-3). 
El desarrollo, crecimiento, alcance e impacto del programa se debe a un compromiso colectivo incomparable. Este, tiene líderes múltiples, tanto dentro de la RNAO, como fuera de nuestra institución. Dentro de la RNAO, existen líderes que, a través de los años, han trabajado conmigo arduamente para asegurar que las guías de buenas prácticas sean desarrolladas siguiendo los más altos cánones internacionales. Así también son los estándares del programa creado por RNAO para la implementación de las guías y la evaluación de su impacto en pacientes y organizaciones sanitarias y académicas que las implementan. Externos a RNAO, si bien muy entrelazados, están los Best Practice Spothlight Organizations -BPSO (por sus siglas en inglés). Estos centros comprometidos con la excelencia, guiados por grandes líderes locales de enfermería y empoderados por sus Champions, son el motor que le da vida al programa. Sus resultados, en diferentes contextos, son reportados por 1,000 instituciones a través del mundo, y demuestran que lo que hace 20 años fue una aspiración, es el día de hoy una realidad.

Este fuerte y mágico liderazgo dentro de cada país se vislumbra claramente en la paginas próximas a leer en este volumen 23(1) de MedUNAB. En este encontrarán un artículo de reflexión derivado de investigación en el que se presenta la implementación curricular de las prácticas clínicas de enfermería en la valoración y selección de dispositivos de acceso vascular, (RNAO), por el docente de la UNAB, Hendrik Adrián Baracaldo Campo. Él resalta el uso del panorama competitivo y permite identificar que el $44.4 \%$ de los cursos del componente básico de la malla curricular del programa y el 38.8\% de los cursos del componente especifico, presentan un impacto mayoritariamente de recomendaciones para la práctica, formación y la organización dentro del currículo para el caso de la guía de dispositivos de acceso vascular. Las colegas Lina María Granados Oliveros, Maribel Esparza Bohorquez, líderes del BPSO - FOSCAL, resaltan sus éxitos alcanzados en el artículo de implementación y sostenibilidad de las guías de enfermería basadas en la evidencia: modelo de la RNAO, haciendo énfasis en los resultados de adherencia y sostenibilidad de tres guías en una institución de tercer nivel, en el que se identifica que la auditoría y verificación periódica de las estrategias implementadas promueve la adherencia del personal de los servicios en el cumplimiento de las metas institucionales y la sostenibilidad del cambio logrado.

Las colegas Cynthia Coltters-Miranda y Alejandra Belmar-Valdebenito de Clínica Las Condes de Santiago de Chile, en su artículo experiencia de implementación de una guía para la prevención de caídas como trabajo interdisciplinar en una unidad de cuidados del adulto mayor, describen cómo el proceso de trabajo interdisciplinario genera disminución de este evento adverso y reduce las caídas con posterior lesión. Por otra parte, los compañeros de España presentan su artículo sobre el rol que juegan las redes sociales en la estrategia de implementación de evidencias en la práctica clínica; su experiencia en el Hospital Regional Universitario de Málaga, España es relatada por Jesús Bujalance Hoyos, Doris Grinspun, María Teresa Pérez Jiménez, Cipriano Viñas Vera, María Soledad Jiménez Fernández y Juan Antonio García Sánchez. Ellos resaltan las posibilidades que nos dan en el uso de las redes sociales. Nos muestran como ya dentro de su institución el uso de las redes sociales ha obtenido unos resultados excelentes de adherencia de los profesionales al programa tanto a nivel cuantitativo como cualitativo.

Colegas: desde su inicio nuestros planes fueron compartir, enriquecer y apasionar a los profesionales de Enfermería a una práctica basada en evidencia para así optimizar los resultados clínicos y de salud de sus pacientes. Al día de hoy, son equipos de salud en el mundo entero los que se van enriqueciendo con este programa y a su vez enriqueciendo el programa mismo. ¡Juntos, estamos construyendo un mundo mejor y más sano para todos!

Tanto en su comienzo, como en el futuro, el programa seguirá centrado en las personas y las comunidades a las que, como enfermeras y como equipos interdisciplinarios, cuidamos. Ellos son los mayores ganadores y jueces de nuestro esfuerzo.

\section{Referencias}

1. Grinspun D. Transforming Nursing Through Knowledge: The Conceptual and Programmatic Underpinnings of RNAO's BPG Program. In D. Grinspun and I. Bajnok (Eds.). Transforming Nursing Through Knowledge: Best Practices for Guideline Development, Implementation Science, and Evaluation Indianapolis, IN, USA: Sigma Theta Tau International Honor Society of Nursing; 2018. 3-27p.

2. Grinspun D. Experiencia obtenida con la herramienta Indicadores de Calidad de Enfermería para Informes y Evaluación NQuIRE distribución. MedUNAB [Internet] 2018; 20(3): 30-31 suplemento. [citado 29 de febrero de 2020]. Recuperado en : https://revistas.unab.edu.co/index.php/ medunab/issue/view/243/Vol.20.N\%C3\%BAmero.3$\underline{\text { Suplemento-2018 }}$

3. Grinspun D. and I. Bajnok. Transforming Nursing Through Knowledge: Best Practices for Guideline Development, Implementation Science, and 
Evaluation Indianapolis, IN, USA: Sigma Theta

Tau International Honor Society of Nursing; 2018.

3-27p.

\section{PhD, MSN, BScN, RN, LLD (hon), Dr (he), FAAN, \\ (D) Doris Grinspun,
on), Dr (hc), FAAN,}

* Registered Nurse, Master of Science in Nursing

Doctor of Philosophy, Doctor of Law (hon), Doctor honouris causa,

Chief Executive Officer, Registered Nurses' Association of

Ontario (RNAO)

E-mail. DGrinspun@rnao.org 